\title{
BMJ Open Decreasing ICU-associated Clostridioides difficile infection through fluoroquinolone restriction, the FIRST trial: a study protocol
}

\author{
Nasia Safdar (D) , ${ }^{1,2}$ Vishala Parmasad (D) , ${ }^{2}$ Roger Brown, ${ }^{3}$ Pascale Carayon, ${ }^{4}$ \\ Alexander Lepak, ${ }^{2}$ John C O'Horo, ${ }^{5}$ Lucas Schulz ${ }^{6}$
}

To cite: Safdar N,

Parmasad V, Brown R, et al. Decreasing ICU-associated Clostridioides difficile infection through fluoroquinolone restriction, the FIRST trial: a study protocol. BMJ Open 2021;11:e046480. doi:10.1136/ bmjopen-2020-046480

- Prepublication history for this paper is available online. To view these files, please visit the journal online (http://dx.doi. org/10.1136/bmjopen-2020046480).

Received 04 November 2020 Accepted 16 June 2021

Check for updates

(C) Author(s) (or their employer(s)) 2021. Re-use permitted under CC BY-NC. No commercial re-use. See rights and permissions. Published by BMJ.

For numbered affiliations see end of article.

Correspondence to

Dr Nasia Safdar;

ns2@medicine.wisc.edu

\section{ABSTRACT}

Introduction Clostridioides difficile infection (CDI) is one of the most common healthcare-associated infections in the USA, having high incidence in intensive care units (ICU). Antibiotic use increases risk of $\mathrm{CDI}$, with fluoroquinolones (FQs) particularly implicated. In healthcare settings, antibiotic

stewardship (AS) and infection control interventions are effective in CDI control, but there is little evidence regarding the most effective AS interventions. Preprescription authorisation (PPA) restricting FQs is a potentially promising AS intervention to reduce CDI. The FQ Restriction for the Prevention of CDI (FIRST) trial will evaluate the effectiveness of an FQ PPA intervention in reducing CDI rates in adult ICUs compared with preintervention care, and evaluate implementation effectiveness using a human-factors and systems engineering model.

Methods and analysis This is a multisite, steppedwedge, cluster, effectiveness-implementation clinical trial. The trial will take place in 12 adult medicalsurgical ICUs with $\geq 10$ beds, using Epic as electronic health record (EHR) and pre-existing AS programmes. Sites will receive facilitated implementation support over the 15-month trial period, succeeded by 9 months of follow-up. The intervention comprises a clinical decision support system for FQ PPA, integrated into the site EHRs. Each ICU will be considered a single site and all ICU admissions included in the analysis. Clinical data will be extracted from EHRs throughout the trial and compared with the corresponding pretrial period, which will constitute the baseline for statistical analysis. Outcomes will include ICU-onset CDI rates, FQ days of therapy (DOT), alternative antibiotic DOT, average length of stay and hospital mortality. The study team will also collect implementation data to assess implementation effectiveness using the Systems Engineering Initiative for Patient Safety model.

Ethics and dissemination The trial was approved by the Institutional Review Board at the University of Wisconsin-Madison (2018-0852-CP015). Results will be made available to participating sites, funders, infectious disease societies, critical care societies and other researchers.

Trial registration number NCT03848689.

\section{Strengths and limitations of this study}

- The FIRST trial will provide one of the few national, multisite, comprehensive studies that investigate the effect on intensive care unit-associated Clostridioides difficile infection (CDI) of fluoroquinoIone preprescription authorisation integrated as a computerised decision support tool.

- Our trial design will allow us to look at changes in outcome measures over time at the same site, delineating a temporal sequence to intensive care unitassociated and hospital-associated CDI, providing more evidence for causality.

- Our approach simultaneously introduces antibiotic stewardship fluoroquinolone prescribing best practices and assesses the introduction of these practices, facilitating continuous implementation improvement.

- The primary limitation to this trial is a slowdown in recruitment rates with the SARS-CoV-2 COVID-19 pandemic and the uncertain effects of this pandemic on current intensive care unit sites.

\section{INTRODUCTION}

\section{Background and rationale}

Clostridioides difficile infection (CDI) is one of the most prevalent healthcare-associated infections in the $\mathrm{USA}^{1}$ and CDI rates are consistently higher in intensive care unit (ICU) settings. ${ }^{2}$ CDI represents a serious threat to patient safety, ${ }^{3}$ and excess costs to acute care hospitals in the USA are estimated to be $\$ 4.8$ billion annually. ${ }^{4}$ Antibiotics are among the most commonly prescribed medications in ICUs and antibiotic exposure is the primary risk factor for CDI ${ }^{5-7}$ This is due to the intestinal dysbiosis caused by antibiotics, particularly broadspectrum agents, ${ }^{78}$ rendering individuals more vulnerable to $\mathrm{CDI}^{7}$

Antibiotic stewardship (AS) interventions are essential to reducing the burden of CDI. ${ }^{9-12}$ The goals of AS are to enhance 
patient outcomes and reduce the inappropriate use and overprescribing of antibiotics. ${ }^{13}$ An analysis of national data indicated that reducing prescription of broadspectrum antibiotics by an estimated $30 \%$ would prevent $26 \%$ of CDI related to inpatient antibiotic use. ${ }^{11}$ This would require only a $5 \%$ reduction of overall antibiotic use. $^{11}$

While there is considerable literature to support the use of infection prevention interventions for reducing $\mathrm{CDI},{ }^{14}$ there remain gaps about the impact and implementation of AS interventions specific to CDI. Existing research has yielded unclear and sometimes conflicting results regarding the impact of AS interventions on CDI rates. ${ }^{14-22}$ Moreover, data on patient outcomes in response to AS interventions are inconsistently defined and limited. ${ }^{1521}$ For these reasons, further evaluation is needed to better understand which specific AS interventions will have the greatest impact on CDI rates. ${ }^{14} 15$ Potential AS strategies promising for CDI reduction include preprescription authorisation (PPA) and postprescription review and feedback. ${ }^{15} 16$ 22-34

Of the antibiotic classes, fluoroquinolones (FQs) are one of the most frequently used in inpatient acute care facilities, where they are prescribed to $16.2 \%$ of patients. ${ }^{35} \mathrm{FQ}$ usage markedly increases the risk of $\mathrm{CDI},{ }^{27-30} 36$ and reductions in FQ use are associated with decreased healthcare facility-onset CDI (HO-CDI) rates in US acute care hospitals. ${ }^{37}$ Rising CDI rates in US hospitals can in part be attributed to the FQ-resistant strain $027 / \mathrm{BI} / \mathrm{NAP} 1{ }^{3}$ which accounts for the largest proportion of HO-CDI cases nationally $(30.7 \%) .^{3}$

\section{Study outcomes and measures}

The trial described in this protocol is designed to implement an FQ PPA intervention and evaluate its implementation effectiveness and impact on CDI rates in adult medical-surgical ICU settings. This approach was chosen because restrictive AS interventions like PPA are likely to be effective; however, implementation is often complex and variable between studies, making implementation evaluation difficult. We propose the integration of an FQ PPA into the electronic health record (EHR) using clinical decision support (CDS) technologies. CDS technologies have demonstrated improvements in patient outcomes in a variety of healthcare settings. ${ }^{38-40}$ We hypothesise that this FQ PPA intervention will result in decreased CDI rates during the intervention period and that quality improvement efforts will be enhanced by the University of Wisconsin-Madison study team external implementation facilitation at each site.

The primary objective is to evaluate the effectiveness of this FQ PPA intervention in reducing ICU-onset and healthcare facility-onset CDI rates in adult ICUs compared with usual care. The secondary objective is to evaluate the effectiveness of the implementation of this intervention using the Systems Engineering Initiative for Patient Safety (SEIPS) model. ${ }^{41}$

\section{METHODS}

\section{Study aims and hypothesis}

The overall hypothesis of this study is that an FQ PPA intervention is an effective strategy to reduce CDI rates in the ICU setting. The primary aim of the trial is to determine the impact of FQ PPA on ICU-onset and HO-CDI rates and other clinical outcomes compared with usual care in medical-surgical adult ICUs enrolled in this trial. Consistent with the STEWARDS (Structured Taskforce of Experts Working at Reliable Standards for Stewardship) panel recommendations, we will collect ICU-onset CDI rates as a subset of HO-CDI rates; HO-CDI rates; and healthcare-associated CDI (HA-CDI) rates as measures of trial effects. ${ }^{42}$ We will also collect antibiotic utilisation data measured in days of therapy (DOT) per patient admission and per patient-days for both FQs and their most common alternatives as primary targets of the intervention.

The secondary aim of the trial is to facilitate and evaluate the implementation process, uptake and effectiveness of the FQ PPA as a complex behavioural intervention using the SEIPS model. ${ }^{41}$ SEIPS provides a broad and flexible way to characterise and evaluate work systems and care processes and the complex relationships among them using five work system elements: people, tools and technologies, tasks, organisational factors, and environmental factors. ${ }^{43}$ This model will be used to characterise and evaluate the AS intervention and its impact on care processes and various patient, organisational and professional outcomes to produce a 'thick' description of implementation processes ${ }^{44-47}$ at each of the sites (described later in this article). These characteristics will then be related to the clinical outcomes of the primary aim in a cross-case analysis. ${ }^{45} 48$

We used the Standard Protocol Items: Recommendations for Interventional Trials reporting guidelines in the preparation of this manuscript. ${ }^{49}$

\section{Overall study design}

A non-randomised, stepped-wedge (NR-SW) cluster design will be used, embedded within an effectivenessimplementation hybrid type 2 trial of ICUs that have elected to implement the FQ PPA. ${ }^{50}$ This design is appropriate as it allows us to simultaneously evaluate the $\mathrm{FQ}$ PPA's clinical effects and the impact of the implementation approach on intervention adoption. As all ICUs were planning to implement $\mathrm{FQ}$ AS interventions for quality improvement practices, the NR-SW wedge design allows each site to receive the trial intervention while serving as its own control, thereby maintaining strong internal validity.

The trial will involve three phases at each ICU site. Phase 1 is a 3-month pre-FQ PPA preparatory period for external facilitation of the implementation, prescriber education, building the FQ PPA CDS best practices alert (BPA), and early contextual and implementation data collection. Phase 2 is the 12-month intervention period during which the FQ PPA-BPA goes live, over which time 


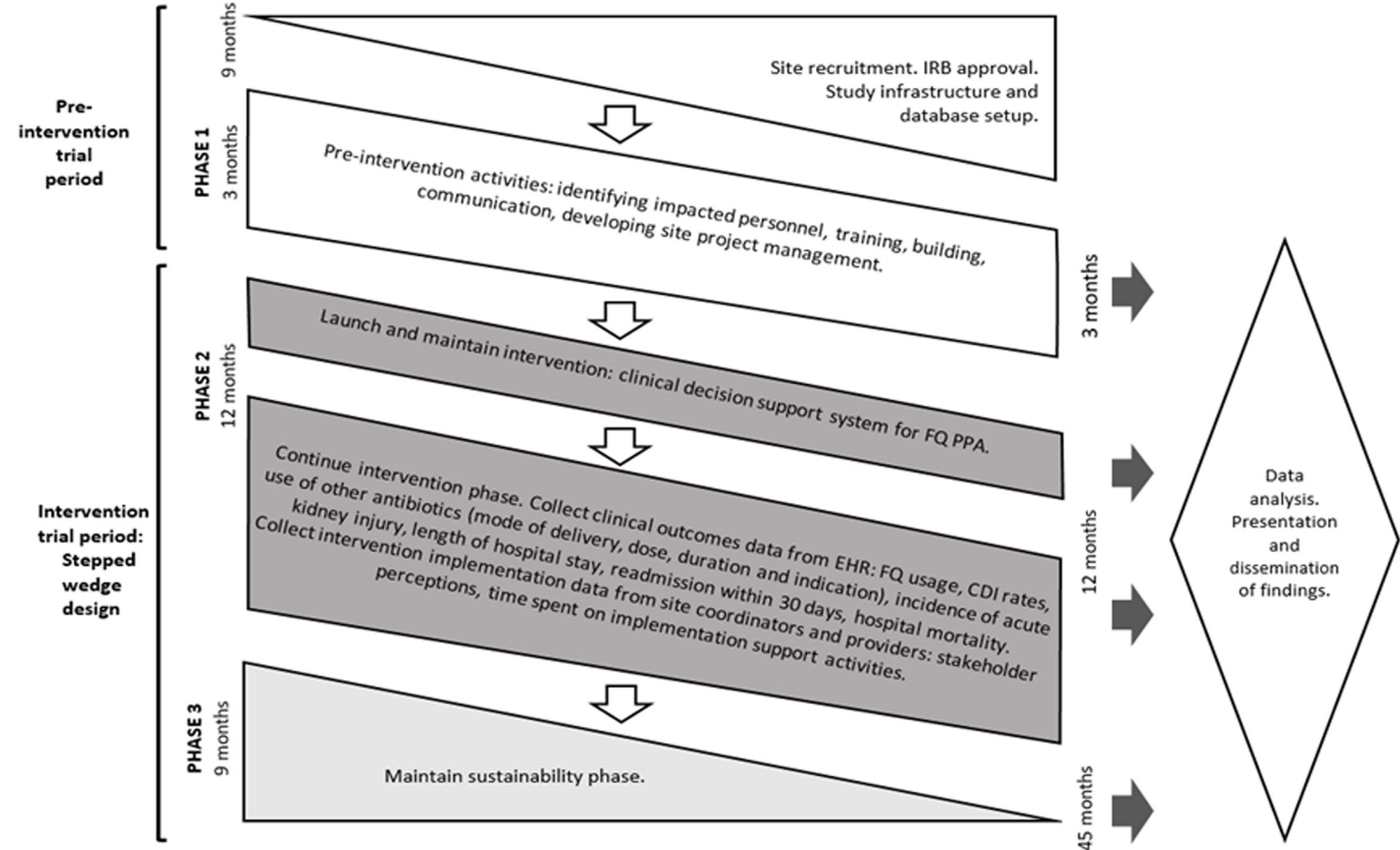

Figure 1 Schematic depiction of the trial design and procedures. CDI, Clostridioides difficile infection; EHR, electronic health record; FQ, fluoroquinolone; IRB, Institutional Review Board; PPA, preprescription authorisation.

both routinely collected clinical EHR data and implementation data will be regularly collected. Phase 3 is a sustainability phase during which sites develop and maintain sustainability action plans and can choose to continue the PPA policy with no further implementation support from the trial team. This sequence will be repeated for each of the sites until all have completed the intervention phase of the trial. Clinical variables and outcomes for the corresponding 12-month preintervention period will constitute the baseline for comparison with the phase 2 intervention period. The influences on implementation and its effectiveness at each site will be assessed using a mixed-methods approach. Figure 1 provides a schematic overview of the study design and method.

\section{Trial organisation}

\section{Steering committee}

The steering committee (SC) will be chaired by the principal investigator (NS) and include the lead biostatistician (RB), coinvestigators (PC, LS, Aurora Pop-Vicas) and other study personnel (VP, AL, Michele Zimbric and Kendra Haight). The SC will meet face-to-face once before study initiation and monthly via teleconference throughout the study. The SC will be responsible for reviewing study progress and if necessary agreeing to protocol changes to facilitate smooth running of the study.

\section{Data coordinating centre}

The data coordinating centre (DCC) will provide expertise and support for the trial in data management, data verification, quality control and assurance, information technology for communication and trial monitoring, and statistical methods for design, including statistical analyses, preparation of results in tabular and graphical formats for presentation, and publication of findings from the trial. The DCC will be located at the University of Wisconsin-Madison, led by the study biostatistician (RB) and the data manager (Fauzia Osman). The University of Wisconsin-Madison team will be responsible for oversight of the DCC activities.

\section{Clinical coordinating centre}

The clinical coordinating centre (CCC) will be responsible for overall study execution: protocol refinement, comprehensive site implementation facilitation, medical monitoring, handling of potential patient-related issues, interfacing with the DCC and coordination with Agency for Healthcare Research and Quality (AHRQ). The CCC will be physically located at the University of WisconsinMadison and led by the principal investigator (NS) and study lead (VP).

\section{Data collection and management}

The electronic case report forms (eCRFs) will be finalised by the DCC before being reviewed and approved by the study team. Data collected at the clinical sites will be de-identified, recorded on eCRFs and entered using the clinical trial data management system. Study investigators will have access to the final trial data set and site personnel will have access to site-specific data.

\section{Site monitoring}

We are planning site virtual initiation visits prior to site enrolment. In addition, we are planning to audit $10 \%$ of 
cases and conduct site audits for cause or on a risk-based priority. All regulatory aspects will be monitored.

\section{Adverse event monitoring}

Adverse event (AE) reporting, such as side effects from alternative antibiotics or inappropriate antibiotic use, will follow established site-specific guidelines for retrospective $\mathrm{AE}$ monitoring and reporting. Existing research on AS interventions, including FQ PPA, indicates that these types of interventions do not have adverse impacts on patient outcomes. While the antibiotics patients receive will be impacted by the FQ PPA intervention, the alternative antibiotics available to providers all fall within best practice guidelines and the possible risks associated with these antibiotics are in equipoise with those associated with FQ. As the purpose of this study is to optimise adherence to established AS best practices, real-time AE monitoring was not considered necessary. Once the study is in place, an independent, ad-hoc drug safety and monitoring board will review a sample of charts from each study site. These charts will be extracted from the study site by site personnel and de-identified before being provided to the University of Wisconsin study team for review.

\section{Patient and public involvement}

The University of Wisconsin team has consistently worked with a patient stakeholder group, the Patients Engaged in Education and Research Group, soliciting feedback regarding patient priorities in healthcare-associated infection prevention. The overall goals of this study are in line with expressed patient priorities of improving AS and decreasing CDI; however, this study specifically targets the prescribing practice of ICU providers. Patients were thus not involved in the design, recruitment, conduct or assessment of the study. The results of this study will be disseminated back to patient stakeholders through venues such as meetings, patient-provider conferences and working with the Madison Patient Education Resource Center.

\section{Study population, inclusion and exclusion criteria}

Adult general medical and surgical ICU sites are the targets of this trial. Participant sites must have a preexisting AS programme with pharmacist and infectious disease physician support and with Epic Systems Corporation as their EHR vendor. Their EHR must have the ability to extract antibiotic usage data (DOT), required outcome data (CDI, mortality, length of ICU stay) and data on indications for antibiotic use. They must additionally be adherent to best practices for infection control relevant to CDI. Sites are considered ineligible to participate if they are already restricting $\mathrm{FQ}$ or another antibiotic associated with risk of CDI. These criteria were selected so that the intervention could be implemented in a standardised manner. The use of Epic Systems Corporation as an EHR vendor was necessary to ensure the changes necessary to the EHR will be feasible at each site. The University of Wisconsin study team will provide templates for and information technology consultations on the required EHR changes and data extraction processes.

Once initiated, the intervention will be applied to all patients admitted to the ICU and all healthcare workers involved in antibiotic prescribing in that ICU. The intervention and usual care strategies will be allocated at the ICU level; thus, inclusion and exclusion criteria apply to ICUs, not to individual patients. Assigning ICUs rather than individuals to the intervention is appropriate given the horizontal transmission of $C$. difficile.

\section{Recruitment and consent}

We chose a total of 12 ICUs to participate in the trial to ensure a patient sample size large enough to detect clinically meaningful and statistically significant differences in CDI outcomes between the intervention and usual care and to account for site attrition. Recruitment emails will be sent out via regional and national research networks, pharmacist networks and AS networks. Informed consent will be obtained by the study lead from all personnel participating in interviews and surveys about implementation and collected data will be de-identified before inclusion in the study. Recruitment will take place on a rolling basis to account for variations in time to completion of pretrial regulatory activities.

\section{Study intervention}

This multicomponent study constitutes a suite of resources for the introduction and assessment of $\mathrm{FQ}$ prescribing best practices in adult ICUs, via an FQ PPA structured around a CDS system within site EHRs. The trial team supports the implementation process at each site and facilitates the development of site-specific CDS FQ PPA protocols.

The FQ PPA CDS intervention constitutes a BPA that appears when providers attempt to prescribe FQs in the ICU. The BPA informs providers that FQ use is restricted and provides links to select alternative antibiotics. Providers can alternatively contact a designated member of the hospital AS team to discuss the choice of drug via the BPA. The BPA and order set will be constructed to allow tracking of non-adherence to the FQ PPA policy, allowing the measurement of fidelity to the intervention. FQs will be discontinued on patients who are already on an FQ when they are transferred to the ICU.

Before and during the implementation of the $\mathrm{FQ}$ PPA policy at each site, the trial team will engage in the external implementation facilitation of this intervention, through supportive activities consistent with evidencebased implementation principles (table 1). ${ }^{51}{ }^{52}$ This approach was purposefully developed by examining relevant implementation literature. ${ }^{52-55}$

\section{Usual care}

Usual care for this trial will include no active restrictions to $\mathrm{FQ}$ use. Sites may still choose to use postprescription feedback for FQ if that is their usual practice. There may be restrictions to other antibiotics as per a site's usual 
Table 1 Evidence-based implementation principles

\begin{tabular}{|c|c|}
\hline $\begin{array}{l}\text { Implementation } \\
\text { principles }\end{array}$ & What will be done at each site \\
\hline $\begin{array}{l}\text { Top } \\
\text { management } \\
\text { commitment }\end{array}$ & $\begin{array}{l}\text { Immediately prior to initiating the PPA, we will ask each site's leadership to communicate support for } \\
\text { the intervention. Depending on the site, this could include the board of directors, medical staff boards of } \\
\text { governance, ICU leadership, ICUs' quality improvement committee, and/or the pharmacy and therapeutics } \\
\text { team. }\end{array}$ \\
\hline $\begin{array}{l}\text { User } \\
\text { participation }\end{array}$ & $\begin{array}{l}\text { After we identify site coordinators, we will ask them to identify the attendings, fellows, residents, advanced } \\
\text { practice providers, pharmacists and ID staff from the AS team who will be impacted by the PPA. }\end{array}$ \\
\hline Training & $\begin{array}{l}\text { We will set up conference calls via webinar with relevant providers in order to provide training. We will have } \\
\text { separate coaching sessions with the unit pharmacists and the AS team to handle calls/questions from } \\
\text { providers regarding FQ prescribing. We will also distribute a toolkit to providers that will include a summary } \\
\text { of research supporting FQ PPA, data on their ICU's CDI and FQ usage rates, an FQ alternative antibiotics } \\
\text { card, a cross-table antibiogram and links to relevant prescribing guides and decision support tools. }\end{array}$ \\
\hline Learning & $\begin{array}{l}\text { Once these activities have been completed, we will closely analyse the barriers and facilitators at each } \\
\text { site and work with site coordinators to address the barriers and leverage facilitators to the greatest extent } \\
\text { possible. Once the PPA policy has been initiated at each site we will continue to provide support to aid the } \\
\text { implementation of the PPA policy. We will also hold monthly phone calls with the site coordinators to discuss } \\
\text { how any emerging barriers can be addressed while maintaining fidelity. }\end{array}$ \\
\hline $\begin{array}{l}\text { Project } \\
\text { management }\end{array}$ & $\begin{array}{l}\text { We will identify coordinators at each site who will act as the primary contact for the trial. We will work with } \\
\text { the coordinators to identify barriers and facilitators for the implementation of the PPA policy at their sites. We } \\
\text { will also ask the coordinators to identify staff who seem enthusiastic about the intervention that may act as } \\
\text { champions at their site. }\end{array}$ \\
\hline
\end{tabular}

AS, antibiotic stewardship; CDI, Clostridioides difficile infection; FQ, fluoroquinolone; ICU, intensive care unit; ID, infectious disease; PPA, preprescription authorisation.

practice and an active AS programme must be in place. Given expected variation in usual practice, we will collect data on usual AS and infection prevention practices at each site to understand the spectrum of usual care.

\section{Data collection and analysis}

Aim 1: data collection

For the primary aim, data will be extracted from each site's Clarity database derived from the PennChart (Epic) EHR application. The trial team will provide each site with a standardised data extraction manual and Microsoft SQL coding-logic document delineating the required data variables. Routinely collected, patient-level clinically generated data will be extracted for the 12-month phase 2 intervention period and the corresponding 12-month preintervention period.

We will collect incidence of HO-CDI, location-specific ICU-onset CDI and HA-CDI. In order to more closely associate the effects on CDI rates with a site's antibiotic use, the fidelity of the intervention will be confirmed by measuring FQ and other antibiotic usage in DOT per patient admission and DOT per 1000 patient-days. To evaluate both the positive and negative clinical outcomes of this intervention to participating ICUs, mortality, readmissions, hospital length of stay and the incidence of other (non-CDI) healthcare-associated infections will also be assessed. Table 2 shows the data variables that will be collected. The de-identified clinical data will be sent to the trial team via a personal health information secure website for statistical analysis.

\section{Aim 1: statistical analysis}

Using 10.5 per 10000 patient-day CDI rate as the base value, reducing it by $50 \%$ based on the literature, and using an NR-SW cluster design, we will need monthly assessments, CDI 12 months preintervention and 12 months postintervention, assuming 10 beds per ICU in 6 ICUs to achieve power at around 0.80 , with two-tailed alpha test at 0.05 . We have selected a far more conservative sample size of 12 ICUs to detect an effect of less than $50 \%$, which may nevertheless be clinically meaningful, also allowing for ICU attrition. Simulation studies ${ }^{56}$ have indicated that adequate power to detect effects in balanced data series, as few as 12 data points, may be reasonable for our regression discontinuity analysis in detecting programme intervention level and trend change.

Multiple ICU units (12 ICUs) will be nested in five hospitals. This would typically provide a very small number of units to be modelled at a hospital level, with not enough data to properly estimate the model. Therefore, we do not plan to establish a hospital-level variable to attempt to account for this clustering. Hospitals as well as ICU type will be included as a covariate. 
Table 2 Variables to be collected for aim 1 analysis

\begin{tabular}{|c|c|c|c|}
\hline Unit-level (or hospital-level) variables & Type of variable & Operational definition & How data are extracted \\
\hline $\begin{array}{l}\text { Healthcare facility-onset CDI with ICU } \\
\text { onset }\end{array}$ & Primary outcome & $\begin{array}{l}\text { Positive test for CDI from } \\
\text { ICU specimen sent from a } \\
\text { symptomatic patient on or } \\
\text { after day } 4 \text { of admission to } \\
\text { healthcare facility. }^{63}\end{array}$ & $\begin{array}{l}\text { Routinely collected by } \\
\text { infection control. }\end{array}$ \\
\hline Healthcare facility-onset CDI & Primary outcome & $\begin{array}{l}\text { Positive test for CDI from a } \\
\text { symptomatic patient on or } \\
\text { after day } 4 \text { of admission to } \\
\text { healthcare facility. }{ }^{63}\end{array}$ & $\begin{array}{l}\text { Routinely collected by } \\
\text { infection control. }\end{array}$ \\
\hline Healthcare-associated CDI & Primary outcome & $\begin{array}{l}\text { Positive test for CDI from a } \\
\text { symptomatic patient who was } \\
\text { discharged from the facility } \leq 4 \\
\text { weeks prior to date of stool } \\
\text { specimen collection. } 63\end{array}$ & $\begin{array}{l}\text { Routinely collected by } \\
\text { infection control. }\end{array}$ \\
\hline $\mathrm{AKI}$ & Secondary outcome & KDIGO guideline definition. ${ }^{64} \dagger$ & EHR via chart review. \\
\hline Mortality & Secondary outcome & Hospital mortality. & Administrative data. \\
\hline Length of stay & Secondary outcome & Duration of stay in the hospital. & Administrative data. \\
\hline Readmissions & Secondary outcome & Within 30 post discharge. & Administrative data. \\
\hline $\begin{array}{l}\text { Other HAls (central line-associated } \\
\text { bloodstream infection) }\end{array}$ & Secondary outcome & During ICU or hospital stay. & $\begin{array}{l}\text { Routinely collected by } \\
\text { infection control. }\end{array}$ \\
\hline Infection control interventions & Descriptive & $\begin{array}{l}\text { Compliance with environmental } \\
\text { cleaning, hand hygiene and } \\
\text { contact precautions. }\end{array}$ & $\begin{array}{l}\text { Routinely collected by } \\
\text { infection control with } \\
\text { direct observations. }\end{array}$ \\
\hline
\end{tabular}

ICUs and associated facilities

\section{Patient-level variables}

Age

\begin{tabular}{|c|c|c|c|}
\hline Race & Descriptive & $\begin{array}{l}\text { American Indian or Alaska } \\
\text { Native; Asian; black or African } \\
\text { American; Native Hawaiian or } \\
\text { other Pacific Islander; whiteł. }\end{array}$ & Extracted from EHR. \\
\hline Comorbidity and severity score & Descriptive & $\begin{array}{l}\text { Charlson Comorbidity Index } \\
\text { score }^{6566} \text { and APACHE score. } \\
68\end{array}$ & Extracted from EHR. \\
\hline Number of prior CDI & Descriptive & $\begin{array}{l}\text { Number of prior cases of } \\
\text { healthcare-associated CDI, } \\
\text { confirmed by positive test. }\end{array}$ & Extracted from EHR. \\
\hline Appropriateness of antibiotic use & Secondary outcome & $\begin{array}{l}\text { Use is concordant with } \\
\text { institutional guidelines as } \\
\text { judged by } 2 \text { AS team members } \\
\text { at each site. }{ }^{69} \text { A physician from } \\
\text { the investigative team (NS) will } \\
\text { adjudicate disagreements } \S \text {. }\end{array}$ & $\begin{array}{l}\text { Chart review of a sample } \\
\text { of cases. }\end{array}$ \\
\hline
\end{tabular}

Descriptive Years.

Extracted from EHR. Male, female, unknown/not Extracted from EHR. provided. 
Table 2 Continued

\begin{tabular}{llll}
\hline Unit-level (or hospital-level) variables & Type of variable & Operational definition & How data are extracted \\
\hline Historical factors & Descriptive & $\begin{array}{l}\text { Historical factors that may } \\
\text { influence findings. }\end{array}$ & $\begin{array}{l}\text { Infection control and AS } \\
\text { data. }\end{array}$ \\
\cline { 2 - 2 } SARS-CoV-2 (COVID-19) infection status & Descriptive & Positive/negative status. & Extracted from EHR. \\
\hline
\end{tabular}

*A single DOT will be recorded for each individual antibiotic administered to a patient on a given day. Antibiotic use will be normalised to patients' DOT per 1000 PD as well as per patient admission.

†The KDIGO guideline defines AKI as any of the following: increase in serum creatinine by $\geq 0.3 \mathrm{mg} / \mathrm{dL}$ within 48 hours, or increase in serum creatinine to $\leq 1.5$ times baseline, or urine volume $<0.5 \mathrm{mg} / \mathrm{kg} / \mathrm{hour}$ for 6 hours. ${ }^{64}$

$\ddagger$ These categories are consistent with the US Office of Management and Budget (OMB) minimum standards for maintaining, collecting and presenting race and ethnicity for all grant projects defined in OMB Directive No 15. The National Institutes of Health Grants Policy Statement supports the use of these categories. ${ }^{70}$

§The following published guidance will be used to judge appropriateness: the Hopkins 'Four Moments in Antibiotic Decision-Making' approach: (1) Was antibiotic therapy indicated based on known clinical, microbiological, radiographic and severity of illness findings of the patient? (2) Was the most appropriate empiric antibiotic regimen selected? (3) Was therapy appropriately adjusted or stopped after a reassessment by day 3 of antibiotics? (4) Was the duration of therapy appropriate for the infection being treated? ${ }^{71}$ Given the intensive resources required for this endeavour, we will focus on sepsis treatment.

AKI, acute kidney injury; APACHE, Acute Physiology and Chronic Health Evaluation; AS, antibiotic stewardship; CDI, Clostridioides difficile infection; DOT, days of therapy; EHR, electronic health record; FQ, fluoroquinolone; HAI, healthcare-associated infection; ICU, intensive care unit; KDIGO, Kidney Disease Improving Global Outcomes; PD, patient-days.

We will use two analytic strategies, the first being a multilevel logit random effects model on the incidence of CDI in all ICU sites, following procedures suggested by Huynh $e$ t $a l \mathrm{~s}^{50}$ simulation for analysis of NR-SW designs. All models will be constructed using MLwiN V.3.02 software. ${ }^{57}$

The second analytic approach will be to use interrupted time series analysis ${ }^{58}$ for step-by-step CDI rates per ICU, using the 12-month preintervention and 12-month postintervention data. In this design, data are collected at multiple instances over time before and after an intervention is introduced to detect whether the intervention has an effect significantly greater than the underlying secular trend. Since we anticipate an abrupt and permanent change in the outcome after implementation of the intervention programme, we propose regression discontinuity analysis using an autoregressive regression model. All interrupted time series models will be constructed using Stata's V.14 routine interrupted time series analysis. ${ }^{59}$

Some sites will be subject to the effects of the COVID-19 pandemic of 2020-2021. Patient-level data about COVID-19 status and percentage of ICU beds occupied by such patients will also be included in the data collection to facilitate analysis of changes to prescribing postpandemic. Since COVID-19 influence is time-varying, incorporation of the time-varying agents into our time series model would be appropriate.

\section{Aim 2: data collection}

Data collection for the implementation evaluation and analysis will occur during phases 1 and 2, simultaneous with intervention launch. Data sources will include (1) aggregated site contextual data (2) implementation process documentation and (3) study feedback from site participants, using Institutional Review Board-approved surveys, semistructured interviews and focus group prompts, and informed consent will be obtained from all participants. See table 3 for a summary of data sources and study outcomes for the secondary aim.

\section{Aim 2: implementation analysis}

The secondary outcome measures of this intervention include evaluating the effectiveness of the implementation processes at each site using the SEIPS conceptual framework. A multiple case study design ${ }^{44} 4560$ with a mixed-methods approach ${ }^{4146} 47$ will be used to evaluate the implementation process, with each participating ICU constituting a single site. The SEIPS framework will be used to relate these characteristics to the effectiveness outcomes at each site in a cross-case analysis (figure 2).

The concurrent implementation of the FQ intervention and evaluation of its impact corresponds to the convergent parallel trial design in mixed-methods research ${ }^{46} 4761$ in which quantitative and qualitative data are collected simultaneously. The final outcome of this analysis will be a 'thick' description of implementation with varying levels of success as measured by the primary outcomes. 'Thick' description refers to the use of qualitative methods that provide depth of understanding of both the process and the inner and outer contexts of intervention implementation, to complement the breadth of understanding allowed by quantitative analysis of clinical data. ${ }^{61}$ Site-specific data will be combined in a cross-case analysis table in an Excel spreadsheet, in an adaptation of the predictor-outcome-consequences matrix of Miles $e t$ $a l .{ }^{48}$ We will use a systematic comparative pattern analysis method to iteratively compare and emphasise the combination of potential contributing factors that function together as a system. ${ }^{60}$ This is an important feature of the analysis that fits with the systems approach, which is at the core of the SEIPS model. ${ }^{41}$ Analysis of the compiled data will be performed by a team of researchers with varied expertise in implementation science, human factors and systems engineering, and infectious disease. 
Table 3 Implementation data sources and analysis

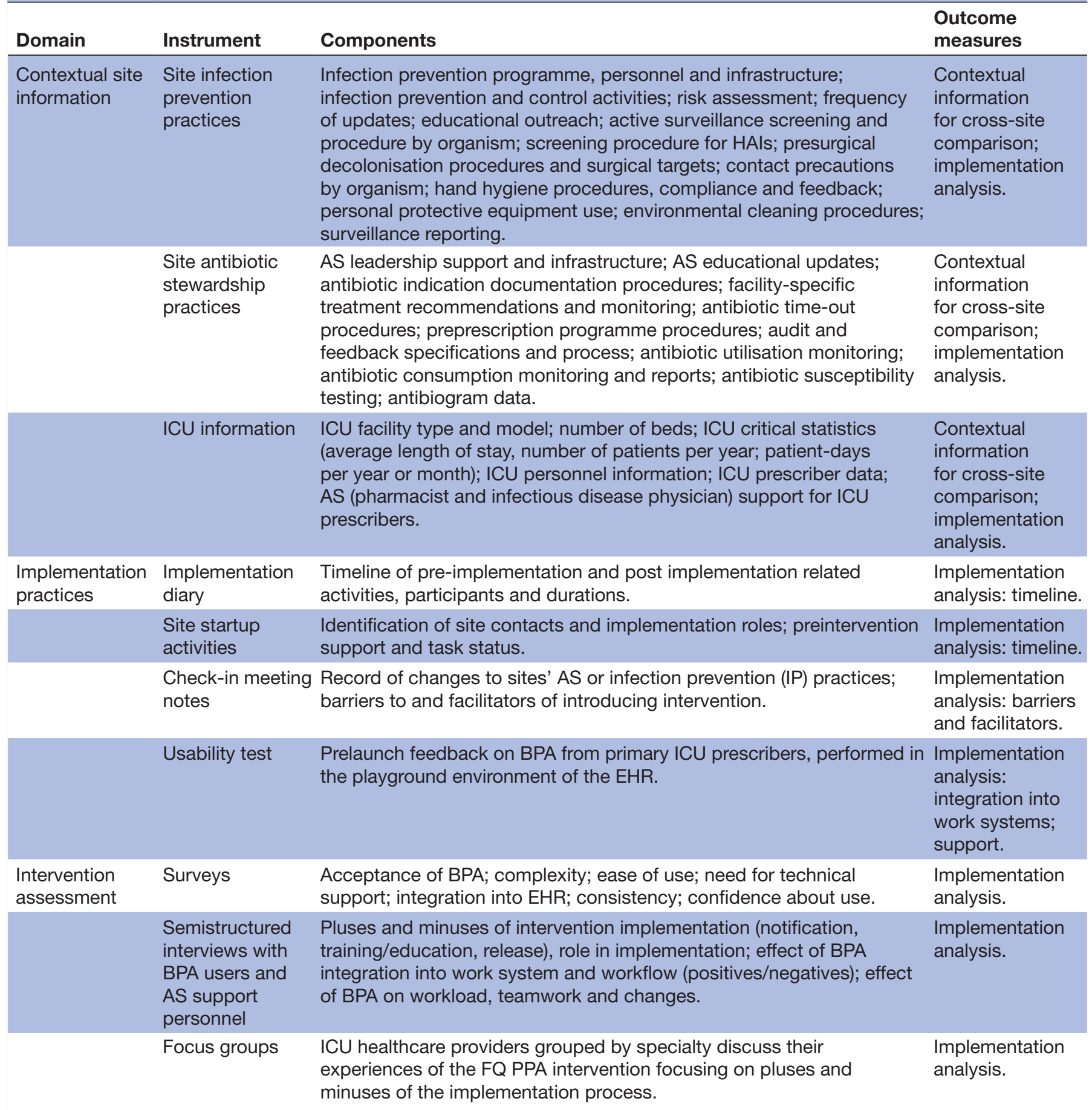

AS, antibiotic stewardship; BPA, best practices alert; EHR, electronic health record; FQ, fluoroquinolone; HAl, healthcare-associated infection; $I C U$, intensive care unit; PPA, preprescription authorisation.

The triangulation with multiple analysts will enhance the quality of the analysis and ensure its rigour. ${ }^{6162}$

\section{DISCUSSION}

We expect this study to demonstrate that the FQPPA intervention results in a decrease in FQ usage in ICU settings and lowers ICU-onset and HO-onset CDI rates. We also expect to have collected rich data on implementation to guide future FQ PPA interventions, including important information on barriers and strategies to overcome them.

At the project conclusion, we will have (1) assessed the effects on CDI rates of the FQ PPA implementationintervention trial and (2) evaluated the most effective implementation processes for introducing this FQ PPA in ICU settings. The knowledge from this project could benefit subsequent projects focused on instituting FQ 


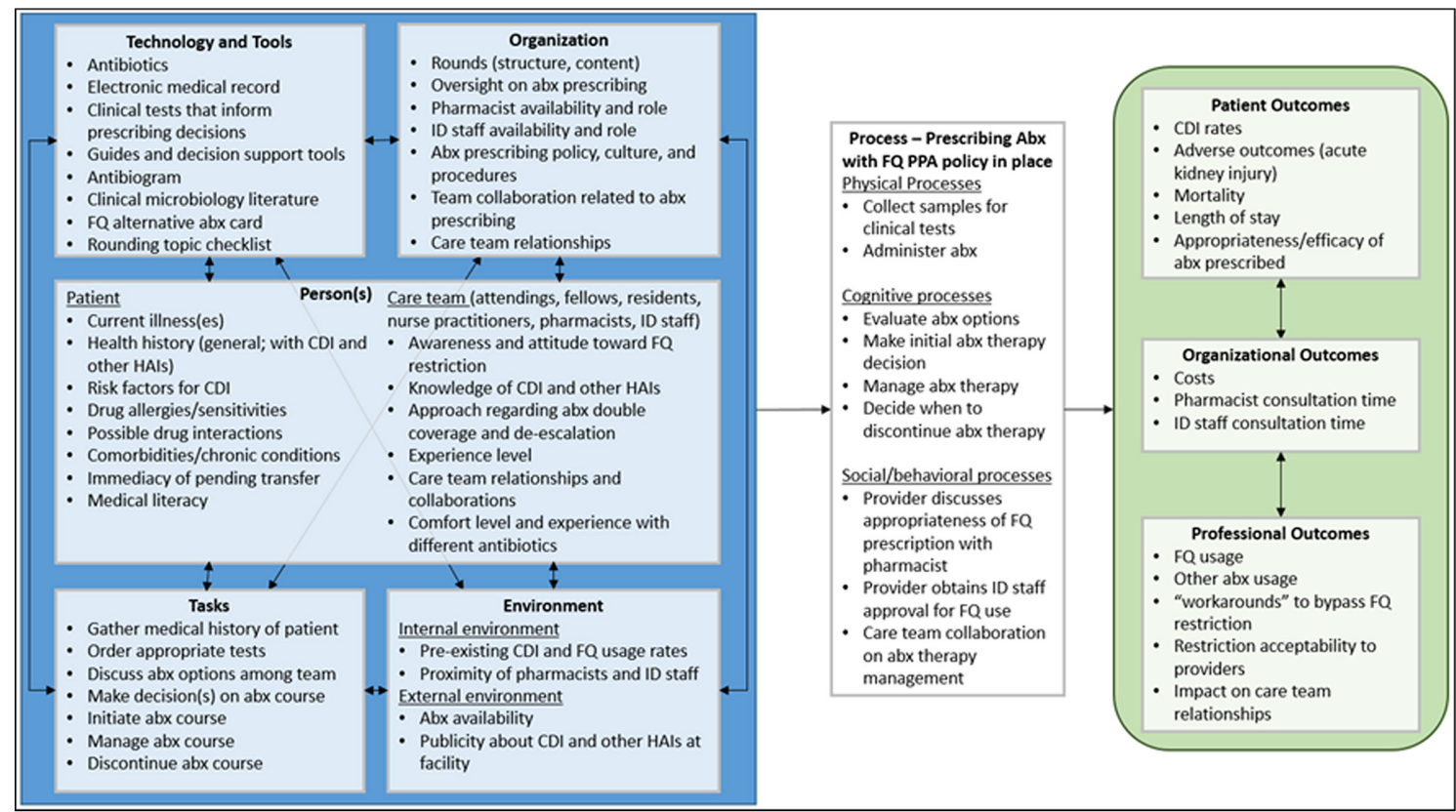

Figure 2 SEIPS framework: FQ PPA implementation in acute care settings. Abx, antibiotics; CDI, Clostridioides difficile infection; FQ, fluoroquinolone; HAI, healthcare-associated infection; ID, infectious disease; PPA, preprescription authorisation; SEIPS, Systems Engineering Initiative for Patient Safety.

PPA in acute care settings and improve the quality of AS programmes nationally. The integration of the FQ PPA into CDS technologies with real-time clinical expertise availability has the potential to improve the quality of antibiotic prescribing throughout the entire hospital systems as well. Given the complexity of this intervention, the findings may not be applicable to the implementation of simpler FQ PPA efforts. However, there are critical gaps in the knowledge of how to best target CDI with AS interventions, which this study will address.

The evolving COVID-19 pandemic of 2020 is likely to affect site recruitment and the results of this trial. Among other effects, prescribing practices for patients with suspected or confirmed COVID-19 infection in the ICU may influence antibiotic use. We will attempt to address this by comparing site prescribing practices preCOVID-19 and post-COVID-19.

\section{Ethics and dissemination}

Ethical approval for this study was obtained from the University of Wisconsin-Madison Health Sciences Institutional Review Board (protocol version: 2018-0852CP015). Individual sites may choose to undergo their own internal review process or cede to the Institutional Review Board of the University of Wisconsin. The study protocol was approved on 24 July 2018 and this manuscript reports on the most updated version of the protocol approved on 19 October 2020. All participant sites will be informed prior to enrolment that participation is completely voluntary, that they can withdraw from participation at any time and that their decision to participate or not will not affect their healthcare in any way.
On completion of the study, we will present the results at major scientific conferences and will publish the results in peer-reviewed journals.

\section{Author affiliations}

${ }^{1}$ Medicine, University of Wisconsin-Madison, Madison, Wisconsin, USA

${ }^{2}$ Medicine, University of Wisconsin-Madison School of Medicine and Public Health, Madison, Wisconsin, USA

${ }^{3}$ School of Nursing, University of Wisconsin-Madison Graduate School, Madison, Wisconsin, USA

${ }^{4}$ Industrial and Systems Engineering, University of Wisconsin-Madison, Madison, Wisconsin, USA

${ }^{5}$ Medicine, Mayo Clinic, Rochester, Minnesota, USA

${ }^{6}$ Pharmacy, University of Wisconsin-Madison School of Medicine and Public Health, Madison, Wisconsin, USA

Contributors NS, PC, RB, AL, JCO and LS conceived of the study concept and design. VP drafted the overall protocol, with critical input from NS and RB for study design, recruitment and statistical analysis. RB drafted the protocol sections for statistical analyses and PC for implementation analysis. All authors provided critical feedback and approved the final version of the manuscript.

Funding This work is supported by grant number R01HS026226 from the Agency for Healthcare Research and Quality and by grant number UL1TR002373 from the Clinical and Translational Science Award (CTSA) program, through the NIH National Center for Advancing Translational Sciences (NCATS). The content and views expressed in this article are solely the responsibility of the authors and do not necessarily represent the position or policy of the Agency for Healthcare Research and Quality and the National Institutes of Health.

Competing interests None declared.

Patient and public involvement Patients and/or the public were not involved in the design, or conduct, or reporting, or dissemination plans of this research.

Patient consent for publication Not required.

Provenance and peer review Not commissioned; externally peer reviewed.

Open access This is an open access article distributed in accordance with the Creative Commons Attribution Non Commercial (CC BY-NC 4.0) license, which permits others to distribute, remix, adapt, build upon this work non-commercially, and license their derivative works on different terms, provided the original work is 
properly cited, appropriate credit is given, any changes made indicated, and the use is non-commercial. See: http://creativecommons.org/licenses/by-nc/4.0/.

\section{ORCID iDs}

Nasia Safdar http://orcid.org/0000-0003-3946-0437

Vishala Parmasad http://orcid.org/0000-0001-8787-4269

\section{REFERENCES}

1 Magill SS, Edwards JR, Fridkin SK, et al. Survey of health careassociated infections. N Engl J Med 2014;370:2542-3.

2 Balsells E, Shi T, Leese C, et al. Global burden of Clostridium difficile infections: a systematic review and meta-analysis. J Glob Health 2019;9:010407.

3 Lessa FC, Winston LG, McDonald LC, et al. Burden of Clostridium difficile infection in the United States. $N$ Engl J Med 2015;372:825-34.

4 Dubberke ER, Olsen MA. Burden of Clostridium difficile on the healthcare system. Clin Infect Dis 2012;55 Suppl 2:S88-92.

5 Bloomfield LE, Riley TV. Epidemiology and risk factors for community-associated Clostridium difficile infection: a narrative review. Infect Dis Ther 2016;5:231-51.

6 Eze $\mathrm{P}$, Balsells E, Kyaw MH, et al. Risk factors for Clostridium difficile infections - an overview of the evidence base and challenges in data synthesis. J Glob Health 2017;7:010417.

7 Crowther GS, Wilcox MH. Antibiotic therapy and Clostridium difficile infection - primum non nocere - first do no harm. Infect Drug Resist 2015;8:333-7.

8 Gerding DN, File TM, McDonald LC. Diagnosis and treatment of Clostridium difficile infection. Infect Dis Clin Pract 2016;24:3-10.

9 Cohen SH, Gerding DN, Johnson S, et al. Clinical practice guidelines for Clostridium difficile infection in adults: 2010 update by the Society for healthcare epidemiology of America (SheA) and the infectious diseases Society of America (IDSA). Infect Control Hosp Epidemiol 2010;31:431-55.

10 Weiner LM, Fridkin SK, Aponte-Torres Z, et al. Vital Signs: Preventing Antibiotic-Resistant Infections in Hospitals - United States, 2014. Am J Transplant 2016;16:2224-30.

11 Fridkin S, Baggs J, Fagan R, et al. Vital signs: improving antibiotic use among hospitalized patients. MMWR Morb Mortal Wkly Rep 2014;63:194-200.

12 Centers for Disease Control and Prevention (CDC). Vital signs: preventing Clostridium difficile infections. MMWR Morb Mortal Wkly Rep 2012;61:157-62.

13 Antimicrobial Stewardship. Society for healthcare epidemiology of America, 2021.

14 Louh IK, Greendyke WG, Hermann EA, et al. Clostridium difficile infection in acute care hospitals: systematic review and best practices for prevention. Infect Control Hosp Epidemiol 2017;38:476-82.

15 Wagner B, Filice GA, Drekonja D, et al. Antimicrobial stewardship programs in inpatient hospital settings: a systematic review. Infect Control Hosp Epidemiol 2014;35:1209-28.

16 Feazel LM, Malhotra A, Perencevich EN, et al. Effect of antibiotic stewardship programmes on Clostridium difficile incidence: a systematic review and meta-analysis. $J$ Antimicrob Chemother 2014;69:1748-54.

17 Davey P, Marwick CA, Scott CL. Interventions to improve antibiotic prescribing practices for hospital inpatients. Cochrane Database Syst Rev 2017;2:Cd003543.

18 Hsu J, Abad C, Dinh M, et al. Prevention of endemic healthcareassociated Clostridium difficile infection: reviewing the evidence. Am J Gastroenterol 2010;105:2327-39.

19 Wenzler E, Mulugeta SG, Danziger LH. The antimicrobial stewardship approach to combating Clostridium difficile. Antibiotics 2015;4:198-215

20 Moffa MA, Walsh TL, Tang A, et al. Impact of an antimicrobial stewardship program on healthcare-associated Clostridium difficile rates at a community-based teaching hospital. $J$ Infect Prev 2018;19:191-4.

21 Moehring RW, Anderson DJ, Cochran RL, et al. Expert consensus on metrics to assess the impact of patient-level antimicrobial stewardship interventions in acute-care settings. Clin Infect Dis 2017;64:377-83.

22 Baur D, Gladstone BP, Burkert F, et al. Effect of antibiotic stewardship on the incidence of infection and colonisation with antibiotic-resistant bacteria and Clostridium difficile infection: a systematic review and meta-analysis. Lancet Infect Dis 2017;17:990-1001.
23 White AC, Atmar RL, Wilson J, et al. Effects of requiring prior authorization for selected antimicrobials: expenditures, susceptibilities, and clinical outcomes. Clin Infect Dis 1997;25:230-9.

24 Buising KL, Thursky KA, Robertson MB, et al. Electronic antibiotic stewardship--reduced consumption of broad-spectrum antibiotics using a computerized antimicrobial approval system in a hospital setting. Journal of Antimicrobial Chemotherapy 2008;62:608-16.

25 Pakyz AL, Oinonen M, Polk RE. Relationship of carbapenem restriction in 22 university teaching hospitals to carbapenem use and carbapenem-resistant Pseudomonas aeruginosa. Antimicrob Agents Chemother 2009;53:1983-6.

26 Metjian TA, Prasad PA, Kogon A, et al. Evaluation of an antimicrobial stewardship program at a pediatric teaching hospital. Pediatr Infect Dis J 2008;27:106-11.

27 Lewis GJ, Fang X, Gooch M, et al. Decreased Resistance of Pseudomonas aeruginosa with Restriction of Ciprofloxacin in a Large Teaching Hospital's Intensive Care and Intermediate Care Units. Infect Control Hosp Epidemiol 2012;33:368-73.

28 Carling $\mathrm{P}$, Fung $\mathrm{T}$, Killion A, et al. Favorable impact of a multidisciplinary antibiotic management program conducted during 7 years. Infect Control Hosp Epidemiol 2003;24:699-706.

29 DiazGranados CA. Prospective audit for antimicrobial stewardship in intensive care: impact on resistance and clinical outcomes. Am J Infect Control 2012;40:526-9.

30 Elligsen M, Walker SAN, Pinto R, et al. Audit and feedback to reduce broad-spectrum antibiotic use among intensive care unit patients: a controlled interrupted time series analysis. Infect Control Hosp Epidemiol 2012;33:354-61.

31 Newland JG, Stach LM, De Lurgio SA, et al. Impact of a Prospective-Audit-With-Feedback antimicrobial stewardship program at a children's Hospital. J Pediatric Infect Dis Soc 2012;1:179-86.

32 Di Pentima MC, Chan S, Hossain J. Benefits of a pediatric antimicrobial stewardship program at a children's Hospital. Pediatrics 2011;128:1062-70.

33 van Kasteren MEE, Mannien J, Kullberg B-J, et al. Quality improvement of surgical prophylaxis in Dutch hospitals: evaluation of a multi-site intervention by time series analysis. J Antimicrob Chemother 2005;56:1094-102.

34 Cosgrove SE, Seo SK, Bolon MK, et al. Evaluation of postprescription review and feedback as a method of promoting rational antimicrobial use: a multicenter intervention. Infect Control Hosp Epidemiol 2012;33:374-80.

35 Talpaert MJ, Gopal Rao G, Cooper BS, et al. Impact of guidelines and enhanced antibiotic stewardship on reducing broad-spectrum antibiotic usage and its effect on incidence of Clostridium difficile infection. J Antimicrob Chemother 2011;66:2168-74.

36 Fridkin SK, Lawton R, Edwards JR, et al. Monitoring antimicrobial use and resistance: comparison with a national benchmark on reducing vancomycin use and vancomycin-resistant enterococci. Emerg Infect Dis 2002;8:702-7.

37 Kazakova SV, Baggs J, McDonald LC, et al. Association between antibiotic use and Hospital-onset Clostridioides difficile infection in US acute care hospitals, 2006-2012: an ecologic analysis. Clin Infect Dis 2020;70:11-18.

38 Garg AX, Adhikari NKJ, McDonald H, et al. Effects of computerized clinical decision support systems on practitioner performance and patient outcomes: a systematic review. JAMA 2005;293:1223-38.

39 Lobach DF, Hammond WE. Computerized decision support based on a clinical practice guideline improves compliance with care standards. Am J Med 1997;102:89-98.

40 Patterson BW, Pulia MS, Ravi S, et al. Scope and influence of electronic health Record-Integrated clinical decision support in the emergency department: a systematic review. Ann Emerg Med 2019;74:285-96.

41 Carayon P, Schoofs Hundt A, Karsh B-T, et al. Work system design for patient safety: the SEIPS model. Qual Saf Health Care 2006;15 Suppl 1:i50-8.

42 Barlam TF, Cosgrove SE, Abbo LM, et al. Implementing an antibiotic stewardship program: guidelines by the infectious diseases Society of America and the Society for healthcare epidemiology of America. Clin Infect Dis 2016;62:e51-77.

43 Holden RJ, Carayon P, Gurses AP, et al. SEIPS 2.0: a human factors framework for studying and improving the work of healthcare professionals and patients. Ergonomics 2013;56:1669-86.

44 Eisenhardt KM. Building theories from case study research. Business and management research methodologies: multi-method research and case studies 2006:218-40.

45 Yin RK. Enhancing the quality of case studies in health services research. Health Serv Res 1999;34:1209-24. 
46 Carayon P, Kianfar S, Li Y, et al. A systematic review of mixed methods research on human factors and ergonomics in health care. Appl Ergon 2015;51:291-321.

47 Creswell JW. Research design : qualitative, quantitative, and mixed methods approaches. 3rd ed. Thousand Oaks, CA: Sage Publications, 2009.

48 Miles MB, Huberman AM, Saldana J. Qualitative Data Analysis - A Methods Sourcebook. 3rd ed. Sage Publications, 2014.

49 Chan A-W, Tetzlaff JM, Gøtzsche PC, et al. Spirit 2013 explanation and elaboration: guidance for protocols of clinical trials. BMJ 2013;346:e7586.

50 Huynh AK, Lee ML, Farmer MM, et al. Application of a nonrandomized stepped wedge design to evaluate an evidencebased quality improvement intervention: a proof of concept using simulated data on patient-centered medical homes. BMC Med Res Methodol 2016;16:143.

51 Karsh B-T. Beyond usability: designing effective technology implementation systems to promote patient safety. Qual Saf Health Care 2004;13:388-94.

52 Xie A, Carayon P, Cox ED. Application of participatory ergonomics to the redesign of the family-centered rounds process. Ergonomics 2015:1-49.

53 Proctor EK, Powell BJ, McMillen JC. Implementation strategies: recommendations for specifying and reporting. Implement Sci 2013;8:139.

54 Powell BJ, Waltz TJ, Chinman MJ, et al. A refined compilation of implementation strategies: results from the expert recommendations for implementing change (ERIC) project. Implement Sci 2015;10:21.

55 Powell BJ, McMillen JC, Proctor EK, et al. A compilation of strategies for implementing clinical innovations in health and mental health. Med Care Res Rev 2012;69:123-57.

56 Zhang F, Wagner AK, Ross-Degnan D. Simulation-based power calculation for designing interrupted time series analyses of health policy interventions. J Clin Epidemiol 2011;64:1252-61.

57 MLwiN Version 2.02. Centre for multilevel modelling, 2005.

58 Lee H, Munk T. Using regression discontinuity design for program evaluation, 2008
59 Linden A. Conducting interrupted time-series analysis for single- and multiple-group comparisons. Stata J 2015;15:480-500.

60 Yin RK. Case study research: design and methods. 2nd ed. Thousand Oaks, CA: Sage, 1994.

61 Dowding D. Best practices for mixed methods research in the health sciences John W. Creswell, Ann Carroll Klassen, Vicki L. Plano Clark, Katherine Clegg Smith for the office of behavioral and social sciences research; qualitative methods overview Jo Moriarty. Qualitative Social Work 2013;12:541-5.

62 Donaldson MS, Mohr JJ. (US) IoM. exploring innovation and quality improvement in health care Micro-Systems: a Cross-Case analysis, 2001.

63 NHSN. Multidrug-Resistant Organism \& Clostridium difficile Infection (MDRO/CDI) Module, 2017.

64 Kellum JA, Lameire N, Aspelin P. Kidney disease: improving global outcomes (KDIGO) acute kidney injury work group. KDIGO clinical practice guideline for acute kidney injury. Kidney International Supplements 2012;2:1-138.

65 D'Hoore W, Sicotte C, Tilquin C. Risk adjustment in outcome assessment: the Charlson comorbidity index. Methods Inf Med 1993;32:382-7.

66 D'Hoore W, Bouckaert A, Tilquin C. Practical considerations on the use of the Charlson comorbidity index with administrative data bases. J Clin Epidemiol 1996;49:1429-33.

67 Knaus WA, Zimmerman JE, Wagner DP, et al. APACHE-acute physiology and chronic health evaluation: a physiologically based classification system. Crit Care Med 1981;9:591-7.

68 Wagner DP, Knaus WA, Draper EA. Statistical validation of a severity of illness measure. Am J Public Health 1983;73:878-84.

69 Tamma PD, Avdic E, Keenan JF, et al. What is the more effective antibiotic stewardship intervention: Preprescription authorization or Postprescription review with feedback? Clin Infect Dis 2017;64:537-43.

70 NIH Grants Policy Statement. National Institutes of health, 2021.

71 The AHRQ Safety Program for Improving Antibiotic Use. Available: https://www.youtube.com/watch?v=M2hvNyhicJQ 